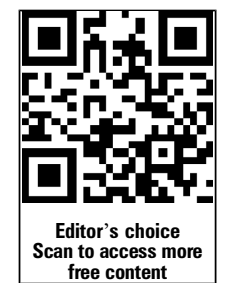

- Additional material is published online only. To view please visit the journal online (http://dx.doi.org/10.1136/ emermed-2013-202965).

${ }^{1}$ Department of Nutrition and Dietetics, Princess Alexandra Hospital, Woolloongabba, Queensland, Australia

${ }^{2}$ Centre for Dietetics Research, School of Human Movements, University of Queensland, St Lucia, Queensland, Australia ${ }^{3}$ Emergency Department, Princess Alexandra Hospital, Ipswich Rd, Woolloongabba, QLD, Australia

${ }^{4}$ Decision Support Team, Princess Alexandra Hospital, Ipswich Rd, Woolloongabba, QLD, Australia

${ }^{5}$ School of Medicine, University of Queensland, Brisbane, Australia

\section{Correspondence to} Dr A Vivanti, Department of Nutrition and Dietetics, Princess Alexandra Hospital, Ipswich Road, Woolloongabba, QLD 4102, Australia; angela_vivanti@health. qld.gov.au

Received 9 July 2013 Revised 24 September 2013 Accepted 26 September 2013 Published Online First

6 November 2013

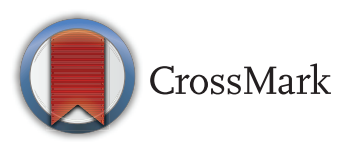

To cite: Vivanti $A$ Isenring $E$, Baumann $S$, et al. Emerg Med 2015;32:180-183

\title{
Emergency department malnutrition screening and support model improves outcomes in a pilot randomised controlled trial
}

\author{
A Vivanti, ${ }^{1,2}$ E Isenring, ${ }^{1,2}$ S Baumann, ${ }^{2}$ D Powrie, ${ }^{1,3}$ M O'Neill, ${ }^{1,4}$ D Clark, ${ }^{1,3}$ \\ $S$ Courtice, ${ }^{1} \mathrm{~K}$ Campbell, ${ }^{1,5}$ M Ferguson ${ }^{1}$
}

\section{ABSTRACT \\ Objective To trial malnutrition screening in older} adults presenting to an emergency department (ED) and compare two service delivery models of nutritional support on nutritional status, quality of life, falls and unplanned hospital admissions.

Design Participants ( $>60$ years) presenting to ED screened at malnutrition risk were randomly allocated to either the control group (receiving regular treatment from community hospital interface programme nursing staff) or intervention group (receiving dietetic assessment, nutrition intervention and follow-up in addition to regular community hospital interface programme support). Outcome measures including body weight, quality of life, depression, falls history and days of hospital admissions were collected at baseline and 12 weeks.

Results of 703 patients screened, 84 (12\%) were identified at malnutrition risk. 24 consented to the intervention study, with $88 \%$ (21/24) confirmed to be malnourished. Clinically important but not statistically significant differences were found over the 12-week trial; the intervention group $(n=9)$ gained $0.8 \mathrm{~kg}( \pm 3.7)$ while the control group $(n=10)$ lost $-1.1 \mathrm{~kg}( \pm 4.6)$. The intervention group also had better quality of life, less depression and shorter hospital admissions.

Conclusions Malnutrition screening appears feasible in ED. This pilot suggests a model of care providing nutrition support to older adults identified at nutritional risk may lead to improved patient outcomes but further research in a larger sample is required to confirm these findings.

\section{INTRODUCTION}

Australia has an ageing population, a finite health budget and proportionally fewer young people to care for the older population in the future. ${ }^{1}$ People aged over 65 years are the fastest growing age group in Queensland ${ }^{2}$ and account for 52\% of hospital admissions in Australia. ${ }^{3}$ Malnutrition is a widespread condition affecting Australia's elderly population and is associated with increased mortality, ${ }^{4} 5$ decreased quality of life $^{6} 7$ and falls risk. ${ }^{8-10}$ Malnutrition has also been associated with increased length of stay among hospitalised patients; ${ }^{11}$ a trend that may be present even among shorter stay acute care units. $^{12}$

Rates of malnutrition in the community have been estimated between $5 \%$ and $20 \%{ }^{13}{ }^{14}$ Preliminary investigation has demonstrated a high prevalence of malnutrition among both older people $(15 \%)$ and frail fallers $(47 \%)$ presenting to emergency departments (ED). ${ }^{15}$ The identification and subsequent treatment of malnutrition is essential for best patient care and outcomes. Timely nutritional support in the elderly population has shown improved outcomes in community or home settings. ${ }^{16-18}$ As hospitalised and malnourished elderly patients commonly fail to meet their energy requirements, nutrition support is paramount for this population. ${ }^{19}$ Evidence-based guidelines emphasise the importance of malnutrition screening across the continuum of care. ${ }^{14}$ The embedding of malnutrition screening within the ED may provide a unique opportunity to identify those community-dwelling older adults who are at nutritional risk.

As ED's are key access points for unwell older people from the community, malnutrition screening in ED's and active nutrition services could substantially reduce the risk of elderly patients being undiagnosed and/or untreated for malnutrition.

The aim of this study was to trial a malnutrition screening programme in older adults admitted to the ED and compare the impact of two service delivery models of nutrition support on nutritional status, quality of life, falls and unplanned hospital admissions.

\section{METHODS}

\section{Research design}

This randomised controlled trial was approved by the Princess Alexandra Hospital Ethics Committee. It included consecutive patients who presented to the ED during Monday to Friday working hours. After providing informed written consent, participants were randomly allocated to either the control or intervention group using a statistician-generated randomised sequence, sealed in sequentially numbered opaque envelopes. The control group received regular treatment through community hospital interface programme nursing staff and community support. The intervention group received nutritional support from an ED dietitian in addition to the community hospital interface programme nursing staff and community support.

Patients eligible for participation in the study were those: presenting to ED; aged 60 years or over; assessed at malnutrition risk using the malnutrition screening tool (MST); ${ }^{20}$ judged by ED staff to be able to provide informed consent or medically stable/suitable to be approached regarding involvement. Patients were excluded if they were: unable to provide informed consent; triage category 1 (highest priority); already receiving dietetic care; admitted from a health care facility (including nursing home). 
Baseline data were collected for all study participants including demographic variables of age and gender. Risk of malnutrition was identified using the MST, which has been shown to have good sensitivity and specificity in the acute geriatric population ${ }^{1421}$ and the ED. ${ }^{15}$ The MST was newly incorporated into the ED admission process for the purposes of the study. The MST consists of two questions pertaining to recent unintentional weight loss and loss of appetite, which generates a numerical score between 0 and 5. Nutritional status was measured using the validated subjective global assessment (SGA) tool ${ }^{22}$ by the ED dietitian.

The number of falls in the past 6 months (including type and severity) was collected through patient self-reports and medical charts. A fall was deemed to occur when a person came to rest inadvertently on the floor, ground or other lower level ${ }^{23}$ as a result of environmental hazards or physical demands exceeding the individual's ability to maintain independent postural stability. ${ }^{24}$ From the patient history, fallers were divided into either the frail mechanical fall group (extensive medical histories and may need ambulatory aids) or active mechanical fall group (falls resulting from active lifestyles or sudden syncope) according to Kingsley. ${ }^{25}$

Quality of life was measured using the EQ-5D tool ${ }^{26}$ and depression was assessed using the global depression score. ${ }^{27}$ One research assistant completed all data collection, eliminating interrater variability. This research assistant received training from a dietitian experienced in nutrition screening and assessment.

\section{Nutrition intervention}

During the 12-week trial, the control group received the standard ED care of assistance from the community hospital interface programme nursing staff in obtaining community support if deemed necessary.

The intervention group received the usual care and also undertook individualised dietary counselling at baseline in which nutrition goals and strategies were made in collaboration with the ED dietitian following standard medical nutrition therapy practice. Follow-up of the intervention was performed at a minimum of weeks 4 and 8 , by telephone review and/or home visit. Final data collection was performed for both intervention and control groups at 12 weeks during a home visit utilising the same baseline assessment tools. In addition, data on death, malnutrition diagnosis, number of presentations to the ED, days of unplanned hospital admissions, and pressure ulcers were recorded from health information management and ED information systems.

\section{Statistical analysis}

All data were assessed using the SPSS for Windows, release V.12.1 (statistical package for the social sciences, 2007). Normally distributed continuous data were presented as means and SD. Data that were not normally distributed are presented as medians and ranges. Categorical data are presented as number and percentages. $\chi^{2}$ and $t$ tests were used with statistical significance determined at $\mathrm{p} \leq 0.05$. The data were also examined to determine clinical significance.

\section{RESULTS}

The study identified that approximately half of the total monthly presentations of older people occurred during Monday-Friday working hours (figure 1). Of the 703 potential study participants screened during the recruiting process, 84 $(11.9 \%)$ were determined to be at risk of malnutrition and hence were invited to join the trial. Twenty-four participants were successfully recruited to the study, 14 of whom were allocated to the control group and 10 to the intervention group. There were four withdrawals (including one death) from the usual care group and one withdrawal in the intervention group.

Participants' mean age was $79.0 \pm 7.7$ years with $42 \%$ male $(10 / 24)$. Of those identified at risk of malnutrition by a score of 2 or more using the MST, $88 \%(21 / 24)$ of participants were confirmed and diagnosed with malnutrition using the SGA. Nine $(n=9)$ participants had presented to ED due to falls.

There were clinically important but not statistically signficant differences between the groups. The intervention group showed an average weight gain of $0.8 \mathrm{~kg}( \pm 3.7)$ compared with weight loss of $-1.1 \mathrm{~kg}( \pm 4.6)$ in the usual care group. The one person whose malnutrition status declined further (rather than maintained or improved) was in the usual care group. All other participants maintained or improved nutritional status as assessed by the SGA. Of the 21 participants assessed as malnourished in the study, only one was captured through the routine hospital data collection, documentation and coding processes.

The intervention group showed improvements in both quality of life and depression in comparison to the control group (table 1). Mean scores of EDQ5 quality of life instrument (0100 scale) improved $14.4 \pm 29$ points with the new model but remained stable among those receiving usual care $(-0.1 \pm 16.4)$.

Median total days of hospitalisation among participants receiving the new model were 4.5 days (range 1-60) compared with a median of 6 days (range 2-59) for the usual care group. Improvement trends may be occurring in the global depression scale scores among the intervention cohort $(-0.9 \pm 3.0)$ in contrast to the control group in which scores moved in a nondesirable direction (1.4 \pm 6.9$)$.

Re-presentations to ED were more frequent among those who, at study end, were assessed as malnourished (1/4) compared with well nourished (1/13). Although six of the nine fallers during the study period were assessed as well nourished at the final data point, fallers were not a homogenous group. Most active fallers $(5 / 6,84 \%)$ were well nourished, while two-thirds $(2 / 3,66 \%)$ of frail fallers were assessed as malnourished.

\section{DISCUSSION}

This study has demonstrated that malnutrition screening in the ED is a feasible method for detecting community-dwelling elderly people who are nutritionally at risk. Malnutrition among older people presenting to ED has been documented at $15 \%$ (one in six) ${ }^{15}$ increasing to $24 \%$ (one in four) among those admitted to hospital after ED presentation. ${ }^{28}$ As not all individuals who present to the ED are admitted to hospital, malnutrition screening in this setting can capture an at-risk population that could otherwise 'slip through the cracks'. The dietetics and ED, with both medical and nursing support, were able to work together collaboratively to incorporate the MST as part of the ED admission process. The tool was successfully implemented and consequently has prompted the redesign of the ED nursing admission documentation to include the MST at our tertiary public hospital.

This pilot study found clinically important but not statistically significant improvements in patient outcomes in those receiving the intervention versus usual care, suggesting the incorporation of malnutrition screening within the ED and dietetic follow-up of patients identified at nutritional risk following admission to the ED may be beneficial. Individuals in the dietetic care model group experienced weight gain compared to weight loss 


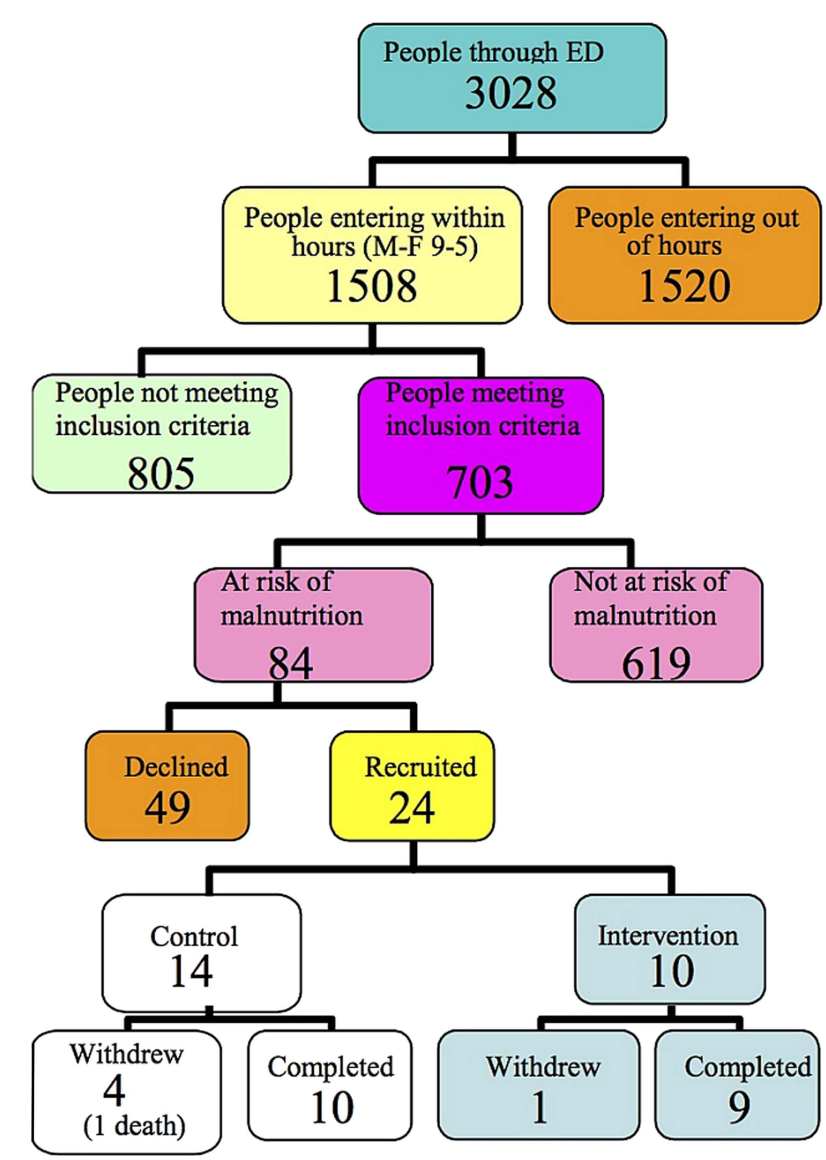

Figure 1 Recruitment of older people aged 60 years or more admitted to emergency departments (ED).

experienced in the control group. For those admitted to hospital, the total days of hospitalisation was shorter for those receiving the new care model.

Nutrition screening and intervention in the ED may improve the management of frail fallers. A large proportion of frail fallers in our pilot study were malnourished when compared with active fallers; who tended to be well nourished. This is consistent with our previous research that found frail fallers were at eight times the risk of being malnourished than active fallers. ${ }^{15}$

Improvements in clinical and health status outcomes from active nutritional support are demonstrated in acute settings. These have included reduced length of stay, ${ }^{29-31}$ reduced

Table 1 Changes in outcome measures on study completion*

\begin{tabular}{|c|c|c|}
\hline Characteristic & $\begin{array}{l}\text { Completed } \\
\text { intervention group } \\
(n=9)\end{array}$ & $\begin{array}{l}\text { Completed } \\
\text { control group } \\
(n=10)\end{array}$ \\
\hline Weight change & $0.8 \mathrm{~kg}( \pm 3.7)$ & $-1.1 \mathrm{~kg}( \pm 4.6)$ \\
\hline Median length of stay (days) & 4.5 (range $1-60$ ) & 6 (range 2-59) \\
\hline EDQ5 quality of life instrument $†$ & $14.4( \pm 29)$ & $-0.1( \pm 16.4)$ \\
\hline Depression $\ddagger$ & $-0.9( \pm 3.0)$ & $1.4( \pm 6.9)$ \\
\hline $\begin{array}{l}\text { Further decline in malnutrition } \\
\text { status }\end{array}$ & $0 / 9$ & $1 / 10$ \\
\hline
\end{tabular}

readmission, increased peak expiratory flow, ${ }^{32}$ improved pressure ulcer ${ }^{29}$ and wound healing. ${ }^{33} 34$ Optimising the nutrition status of those presenting to ED at risk of malnutrition may also result in such outcomes, but is yet to be investigated. Such findings are important to ensure optimal utilisation of health service provision within finite resources.

With the enhanced nutritional support, patient centred outcomes such as quality of life and depression scores were improved with the new service model when compared to the control model. These findings are consistent with improvements found by other authors concerning quality of life ${ }^{33} 35$ and depression $^{36}$ following nutritional interventions. In addition, using EQ-5D and a validated MST, Kvamme et $a l^{37}$ found that increasing malnutrition risk is associated with decreased quality of life scores. Cabrera et $a l^{38}$ similarly found that depression, as measured by the global depression score, was associated with greater malnutrition risk. Our pilot suggests positive shifts are possible following dietetic intervention.

Importantly, this study also identified that malnourished people were not identified or coded through current hospital processes, emphasising the critical role for malnutrition screening in ED. Of the 21 participants assessed as malnourished in the study, only one was captured through routine hospital data collection, documentation and coding processes. The lack of malnutrition identification is consistent with the findings of Agarwal and colleagues, ${ }^{11}$ in which nutrition screening was performed on only $64 \%$ of Australian hospital wards and has implications both on patient outcomes and revenue based on patient acuity.

\section{Strength and limitations}

To the authors' knowledge, this is the first nutrition intervention study conducted within the real world setting of the ED. It used validated measures, involved collaboration from several health disciplines and resulted in improved nutrition screening practice.

The ED proved a difficult environment for participant recruitment due to its dynamic nature and the stress of patients who present in this setting. The sample size of the study was not adequately powered to show statistical significance of the results. Using the effect sizes from this pilot, approximately 50 people in each group would be required for statistically significant results. Further research should therefore endeavour to include a greater sample size to overcome this issue. In addition, many individuals who met the inclusion criteria were preoccupied and apprehensive given the many and varied events at the point of presentation and were dissuaded by the follow-up required given the uncertainty of their future at the time. Due to the challenges presented by the ED setting, shorter nutrition intervention may be a more feasible model to adopt. The model of screening in the ED setting and providing dietetic intervention once the patient has been discharged home through telephone contact may be the most appropriate model for this patient cohort. In addition, providing intervention by telephone after discharge, rather than while the patient is in the ED, aligns with the current context of the national time-based targets for length of stay in ED.

\section{CONCLUSION}

This pilot randomised controlled trial suggests that the ED is a suitable setting for the identification of community dwellers at nutritional risk. Conducting malnutrition screening in ED, including of frail mechanical fallers, might substantially reduce the risk of older patients remaining undiagnosed and untreated 
for malnutrition. This pilot suggests a model of care providing nutrition support to older adults identified at nutritional risk may lead to improved nutrition outcomes, better quality of life and shorter hospitalisations, but these findings need to be confirmed in a larger sample.

Acknowledgements The authors would like to thank the Queensland Health Allied Health Grants, Sarah Anthony and the ED's medical, nursing and allied health staff for enabling this project.

Contributors AV was involved in all aspects of the study, including design, data collection, analysis and interpretation, and writing and editing the manuscript. El, $\mathrm{KC}$ and MF were involved in design, interpreting the data, and in the writing and editing of the manuscript. SB was involved with the writing and editing the manuscript. DP and DC were involved with the original design and operational liaison with ED and staff. MO was involved with the design, data collection and preparing the final manuscript. SC completed data collection, analysis and was involved with interpretation and manuscript completion. All authors critically reviewed the manuscript and approved the final version submitted for publication.

Funding Funding was provided by Queensland Health allied health grants.

Competing interests None.

Ethics approval This randomised controlled trial was approved by the Princess Alexandra Hospital Ethics Committee.

Patient consent Obtained.

Provenance and peer review Not commissioned; externally peer reviewed.

\section{REFERENCES}

1 Australian Institute of Health and Welfare (AIHW) \& Australian Medical Workforce Advisory Committee (AMWAC). Medical workforce supply and demand in Australia: a discussion paper. Canberra: AlHW, 1999.

2 Queensland Government. Queensland 2020: a state for all ages. Department of Families, Brisbane Queensland Government, 2003.

3 Karmel R, Hales C, Lloyd J. Older Australians in hospital. Canberra: AlHW, 2007.

4 Agarwal E, Ferguson M, Banks M, et al. Malnutrition and poor food intake are associated with prolonged hospital stay, frequent readmissions, and greater in-hospital mortality: results from the Nutrition Care Day Survey 2010. Clin Nutr 2013:32:737-45.

5 Isabel TD, Correia M, Waitzberg DL. The impact of malnutrition on morbidity, mortality, length of hospital stay and costs evaluated through a multivariate model analysis. Clin Nutr 2003;22:235-9.

6 Rasheed S, Woods RT. Malnutrition and quality of life in older people: a systematic review and meta-analysis. Ageing Res Rev 2013;12:561-6.

7 Ferguson M, Capra S, Bauer J, et al. Quality of life in patients with malnutrition. J Am Diet Assoc 1998;98(Suppl. 9):A22.

8 Coleman Y, Welsh S, McMahon J. Nutrition and falls. Geriaction 2000;18:12-13.

9 Vellas B, Baumgartner $R$, Wayne $S$, et al. Relationship between malnutrition and falls in the elderly. Nutrition 1992;8:105.

10 Bauer JD, Isenring E, Torma J, et al. Nutritional status of patients who have fallen in an acute care setting. J Hum Nutr Diet 2007;20:558-64.

11 Agarwal $E$, Ferguson $M$, Banks $M$, et al. Nutrition care practices in hospital wards: results from the Nutrition Care Day Survey 2010. Clin Nutr 2012;31:995-1001.

12 Thomas JM, Isenring E, Kellett $E$. Nutritional status and length of stay in patients admitted to an acute assessment unit. J Hum Nutr Diet 2007;20:320-8.

13 Leggo $M$, Banks $M$, Isenring $E$, et al. A quality improvement nutrition screening and intervention program available to home and community care eligible clients. Nutr Diet 2008;65:162-7.

14 Watterson C, Fraser A, Banks M, et al. Evidence based practice guidelines for the nutritional management of malnutrition in adult patients across the continuum of care. Nutr Diet 2009;66:S1-S34.
15 Vivanti AP, McDonald CK, Palmer MA, et al. Malnutrition associated with increased risk of frail mechanical falls among older people presenting to an emergency department. Emerg Med Australas 2009;21:386-94.

16 Bourdel-Marchasson I, Barateau M, Rondeau V, et al. A multi-center trial of the effects of oral nutritional supplementation in critically ill older inpatients. GAGE Group. Groupe Aquitain Geriatrique d'Evaluation. Nutrition 2000;16:1-5.

17 Gray-Donald K, Payette H, Boutier V. Randomized clinical trial of nutritional supplementation shows little effect on functional status among free-living frail elderly. J Nutr 1995;125:2965-71.

18 Collins CE, Kershaw J, Brockington S. Effect of nutritional supplements on wound healing in home-nursed elderly: a randomized trial. Nutrition 2005;21:147-55.

19 Mudge AM, Ross LJ, Young AM, et al. Helping understand nutritional gaps in the elderly (HUNGER): a prospective study of patient factors associated with inadequate nutritional intake in older medical inpatients. Clin Nutr 2011;30:320-5.

20 Ferguson ML, Bauer J, Gallagher B, et al. Validation of a malnutrition screening tool for patients receiving radiotherapy. Australas Radiol 1999;43:325-7.

21 Wu ML, Courtney MD, Shortridge-Baggett LM, et al. Validity of the malnutrition screening tool for older adults at high risk of hospital readmission. J Gerontol Nurs 2012:38:38-45.

22 Detsky AS, McLaughlin JR, Baker JP, et al. What is subjective global assessment of nutritional status? JPEN J Parenter Enteral Nutr 1987;11:8-13.

23 World Health Organization, Geneva. http://www.who.int/ageing/en/ [cited May 2008].

24 Thomas DC, Edelberg HK, Tinetti ME. Falls. In: Cassel CK, Leipzig RM, Cohen RM, et al. eds. Geriatric medicine: an evidence based approach. New York: Springer-Verlag Inc, 2003:979-94.

25 Kingsley S. A breakdown on falls in the elderly. Canadian Journal of Continuing Medical Education 2005:2005:59-61.

26 Shaw JW, Johnson JA, Coons SJ. U.S. validation of the EQ-5D health states development and testing of the D1 valuation model. Med Care 2005;43:203-20.

27 Yesavage JA, Brink TL, Rose TL, et al. Development and validation of a geriatric depression screening scale: a preliminary report. J Psychiatr Res 1982;17:37-49.

28 Potter MA, Luxton G. Prealbumin measurement as a screening tool for protein calorie malnutrition in emergency hospital admissions: a pilot study. Clin Invest Med 1999:22:44-52

29 Stratton RJ, Ek AC, Engfer M, et al. Enteral nutritional support in prevention and treatment of pressure ulcers: a systematic review and meta-analysis. Ageing Res Rev 2005;4:422-50.

30 Koretz R, Avenell A, Lipman T, et al. Does enteral nutrition affect clinical outcome? A systematic review of the randomized trials. Am J Gastroenterol 2007;102:412-29.

31 Avenell $A$, Handoll $H$. Nutritional supplementation for hip fracture aftercare in older people. Cochrane Database Syst Rev 2006;(4):CD001880.

32 Norman $\mathrm{K}$, Kirchner $\mathrm{H}$, Freudenreich $\mathrm{M}$, et al. Three month intervention with protein and energy rich supplements improve muscle function and quality of life in malnourished patients with non-neoplastic gastrointestinal disease-A randomized controlled trial. Clin Nutr 2008:27:48-56.

33 Collins C, Kershaw J, Brockington S. Effect of nutritional supplements on wound healing in home-nursed elderly: a randomized trial. Nutrition 2005;21:147-55

34 Eneroth $\mathrm{M}$, Apelqvist J, Larsson J, et al. Improved wound healing in transtibial amputees receiving supplementary nutrition. Int Orthop 1997;21:104-8.

35 Nijs K, de Graaf C, Kok F, et al. Effect of family style mealtimes on quality of life, physical performance and body weight of nursing home residents: cluster randomised controlled trial. BMJ 2006;332:1180-4.

36 Gariballa S, Forster S. Effects of dietary supplements on depressive symptoms in older patients: a randomised double-blind placebo-controlled trial. Clin Nutr 2007;26:545-51.

37 Kvamme JM, Olsen JA, Florholmen J, et al. Risk of malnutrition and health-related quality of life in community-living elderly men and women: the Tromso study. Qual Life Res 2011;20:575-82.

38 Cabrera MA, Mesas AE, Garcia AR, et al. Malnutrition and depression among community-dwelling elderly people. J Am Med Dir Assoc 2007;8:582-4. 